\title{
Correction: How cultural evolution can inform the science of science communication-and vice versa
}

\section{Theiss Bendixen}

Correction to: Humanities and Social Sciences Communications https://doi.org/10.1057/s41599-020-00634-4, published online 04 November 2020

The original paper contained some typographical errors that have now been corrected as follows:

1. In footnote 6, the original read: "This problematic practice is likelycorroborated [sic] further if cultural linkage is at play and not taken into account". This now reads: "This problematic practice is aggravated further if cultural linkage is at play and not taken into account".

2. In this sentence: "The content properties of a cultural trait influences the likelihood that trait will culturally spread", a "that" was wrongly omitted. This now reads: "The content properties of a cultural trait influences the likelihood that that trait will culturally spread".

3. In footnote 1, the original read: "This three-part partition is not watertight and serve mostly heuristic purposes for the present discussion". This now reads: "This three-part partition is not watertight and serves mostly heuristic purposes for the present discussion".

4. The location of footnote 6 has now been moved to the end of a sentence for clarity.

Open Access This article is licensed under a Creative Commons Attribution 4.0 International License, which permits use, sharing, adaptation, distribution and reproduction in any medium or format, as long as you give appropriate credit to the original author(s) and the source, provide a link to the Creative Commons license,

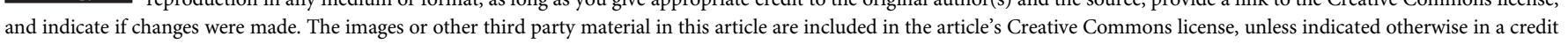

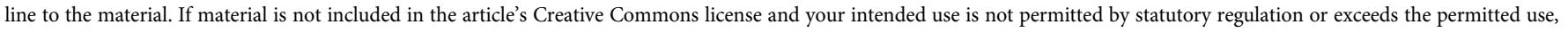
you will need to obtain permission directly from the copyright holder. To view a copy of this license, visit http://creativecommons.org/licenses/by/4.0/.

(C) The Author(s) 2020 